\title{
MIMO Communications in Ad Hoc Networks
}

\author{
Biao Chen, Member, IEEE, and Michael J. Gans, Fellow, IEEE
}

\begin{abstract}
We study in this paper the network spectral efficiency of a multiple-input multiple-output (MIMO) ad hoc network with $K$ simultaneous communicating transmitter-receiver pairs. Assuming that each transmitter is equipped with $t$ antennas and each receiver with $r$ antennas and each receiver implements single-user detection, we show that in the absence of channel state information (CSI) at the transmitters, the asymptotic network spectral efficiency is limited by $r$ nats $/ \mathrm{s} / \mathrm{Hz}$ as $K \rightarrow \infty$ and is independent of $t$ and the transmit power. With CSI corresponding to the intended receiver available at the transmitter, we demonstrate that the asymptotic spectral efficiency is at least $t+r+2 \sqrt{t r}$ nats/s/Hz. Asymptotically optimum signaling is also derived under the same CSI assumption, i.e., each transmitter knows the channel corresponding to its desired receiver only. Further capacity improvement is possible with stronger CSI assumption; we demonstrate this using a heuristic interference suppression transmit beamforming approach. The conventional orthogonal transmission approach is also analyzed. In particular, we show that with idealized medium access control, the channelized transmission has unbounded asymptotic spectral efficiency under the constant per-user power constraint. The impact of different power constraints on the asymptotic spectral efficiency is also carefully examined. Finally, numerical examples are given that confirm our analysis.
\end{abstract}

Index Terms-Ad hoc networks, multiple-input multiple-output (MIMO) communications, spectral efficiency.

\section{INTRODUCTION}

$\mathbf{M}$ ULTIPLE-INPUT multiple-output (MIMO) communications through the use of multiple antenna transceivers have shown great promise in providing spectral efficiencies that are several orders of magnitude higher than that of the traditional communication systems [1]-[3]. There has also been interest in extending the MIMO communication concept to multiple-user systems, most notably in MIMO broadcast [4]-[7] and MIMO multiple access [8], [9] systems. However, both systems have one end of the communication link being centralized - the transmitter in MIMO broadcast and receiver in MIMO multiple access channels. Recently, there is an increasing need for mobile networks with distributed transmitters and receivers, typically referred to as mobile ad hoc networks (MANET) [10],

Manuscript received December 21, 2004; revised August 22, 2005. This work was supported by the Air Force Research Laboratory/Information Institute through the 2004 summer visiting faculty program. Part of this work was presented in the 2005 IEEE International Conference on Acoustic Speech and Signal Processing (ICASSP), Philadelphia, PA, March 2005. The associate editor coordinating the review of this manuscript and approving it for publication was Prof. Alex B. Gershman.

B. Chen is with Department of Electrical Engineering and Computer Science, Syracuse University Syracuse, NY 13244 USA (e-mail: bichen@ecs.syr.edu).

M. J. Gans is with AFRL/IFGC, Rome, NY 13442-4505 USA (e-mail: gansm@rl.af.mil).

Digital Object Identifier 10.1109/TSP.2006.874842
[11]. There, transmitters and receivers do not pool their information together, either due to geographical dispersiveness, the bandwidth and resource limitation, or due to security/privacy concern. It is, therefore, of great interest to study the performance limit of MANET with MIMO transceivers, i.e., when all the nodes are equipped with multiple antennas.

One simple way of utilizing the MIMO potential in MANET is to use channelized transmission, i.e., we partition the total time-frequency space into orthogonal subchannels and allow interference-free MIMO communication in each subchannel. This is hereafter referred to as channelized MIMO (C-MIMO) in the current work. To be able to realize the MIMO spectrum efficiency in a C-MIMO system, one needs to have adaptive channelization that guarantees access to all active users in a way that leaves no idle channels. This is difficult in an ad hoc network with dynamic and distributed user activities due to the lack of a central node (i.e., a base station). On the other hand, recognizing that multiple antennas at the transceivers provide inherent multiplexing capability due to their spatial selectivity, it is attractive to study MIMO communication in ad hoc networks with "interference" transmission (i.e., not channelized). This may alleviate the need for a fully adaptive medium access control (MAC) layer while avoiding spectrum underutilization caused when a fixed channel allocation is used. In addition, by allowing simultaneous transmissions, one can also exploit the multiuser diversity to potentially improve the overall spectral efficiency relative to an idealized C-MIMO system.

In [12], the author studied the MIMO capacity with interference where single-user detection is assumed at the receiver. The results are equally applicable to an ad hoc MIMO network with simultaneous pairwise transmissions. Without knowing the CSI at the transmitter, the author showed that, depending on the interference to noise power ratio, the transmitter should either put equal power into each antenna (optimal in the interference-free MIMO transmission) or operate on a singular mode (i.e., the transmitter puts all power on a single element). In this paper, we establish how the network spectral efficiency, defined as the sum of spectral efficiencies of all active users, scales as the number of transmitting pairs increases. By assuming $t$ transmit and $r$ receive antennas for each transceiver pair, we show that in the absence of CSI at the transmitter and as the number of transmitter-receiver pairs $K$ increases, the total capacity of such an ad hoc interference network is fundamentally limited by the receive antenna size $r$ and is independent of all the other parameters, including $t$ and the transmit power. This results in a per node spectral efficiency of $O(1 / K)$ for fixed $r$, which decreases to 0 as $K \rightarrow \infty$. We call this the "receiver-only CSI" approach.

When the CSI corresponding to the intended receiver is available at the transmitter, we show that a simple "beamforming" 
approach achieves a spectral efficiency of approximately $t+$ $r+2 \sqrt{t r}$ nats $/ \mathrm{s} / \mathrm{Hz}$ for large $t$ and $r$ as $K \rightarrow \infty$; i.e., $t+$ $r+2 \sqrt{t r}$ nats/s/Hz provides a lower bound on the asymptotically achievable spectral efficiency for large $t$ and $r$. For example, with $t=r$, i.e., each transceiver uses the same number of transmit and receive antennas, the total spectral efficiency is $4 r$ nats $/ \mathrm{s} / \mathrm{Hz}$, which is four times higher than that of the channel-blind approach. Nonetheless, the asymptotic per node spectral efficiency still decreases to zero for fixed $t$ and $r$ as the number of pairs $K$ increases. Thus, to achieve nonzero per node spectral efficiency, one needs to scale up $r$ in the absence of CSI at the transmitter, and either $t$ or $r$ in the presence of CSI at the transmitter. This is due the fundamental limit on the multiplexing gain (degree of freedom) imposed by the transmit/receive antenna size [13].

The exact asymptotic optimum spectral efficiency with CSI still remains an open problem. However, we derive the asymptotically optimum transmission scheme which amounts to a waterfilling solution for a composite channel matrix incorporating the interference power. We expect that, with stronger CSI assumptions, better spectral efficiency may result. Toward this end, we assume that a transmitter knows not only the channel to the desired receiver, but also to other receivers it interferes with. We demonstrate through numerical examples that a heuristic interference suppression transmit beamforming approach can achieve better spectral efficiency than the simple beamforming approach with finite number of active users.

Finally, as a comparison, we discuss the asymptotic spectral efficiency for C-MIMO where each pair of users communicate in a subchannel free of interference. Assume idealized MAC, it is straightforward to establish that the network spectral efficiency grows unbounded as more users are added. This is due to the assumption that each user is assigned the same fixed power, which does not vary with the total number of users. Hence, adding more users to the system results in an increased total power. As such, asymptotically the channelized MIMO system will outperform interference transmission in terms of spectral efficiency. However, if a constant total power constraint is assumed for the whole network, a C-MIMO system manifests a constant spectral efficiency which is independent of the number of users.

The ad hoc network model we adopt in the current work employs interference transmissions. This is closely related to the classical inference channel [14], [15]. One obvious difference is that the current work studies MIMO communications in fading channels. A perhaps more important distinction is the assumption of single-user detection at the receiver. This assumption treats all unintended transmissions as pure interference. Therefore, the classical interference channel approach that employs interference cancellation at the receiver does not apply. This single-user detection assumption may be justified by many applications we are interested in: in an ad hoc network involving large numbers of nodes, acquiring CSI from all interfering transmitters incurs an enormous overhead. This overhead, while difficult to quantify, may actually diminish any potential throughput improvement by exploiting the CSI for interference cancellation. Other practical reasons include the security concern: often times a private codebook is shared between each transmitter-receiver pair, thus preventing a receiver from decoding other users' information.

Also related to the current work is the capacity region study of an ad hoc network [11] for a given transmission protocol which consists a set of different transmission schemes. Through the construction of the so-called basic rate matrices, each of them associated with a particular transmission scheme, the achievable rate region is determined numerically by finding the convex hull of the so-called basic rate matrices. This was later extended to the MIMO case [16], where each node is assumed to be equipped with multiple antennas. The framework developed in [11] can easily accommodate multihop communications in ad hoc networks; its complexity, however, makes it difficult to apply to large system analysis.

The rest of the paper is organized as follows. In Section II, we describe the system model. We show in Section III that in the absence of transmitter CSI, the asymptotic network spectral efficiency of a MIMO ad hoc network with interference transmission (the "receiver-only CSI" approach) is fundamentally limited by the number of receive antennas $r$ and is independent of other system parameters. With CSI available at the transmitter, we show in Section IV that a simple "beamforming" approach can improve the spectral efficiency over the blind transmission. Section V gives the spectral efficiency result for C-MIMO systems with ideal MAC. Numerical examples are presented in Section VI where we also demonstrate that with stronger CSI assumptions, better spectral efficiency can be obtained. We conclude in Section VII, where we remark on some future research topics for MIMO MANET.

We use boldface capital letters to denote matrices and boldface lower case letters to denote vectors. $\mathbf{A}^{H}$ is Hermitian (complex conjugate transpose) of $\mathbf{A} .|\mathbf{A}|$ is the determinant of matrix $\mathbf{A}$. I is an identity matrix with appropriate dimension which should be clear in the context it appears. The $\log (\cdot)$ is natural logarithmic function hence the resulting mutual information is in nats instead of bit. While $\mathbf{H}_{k j}$ is used to denote the channel matrix from the $j$ th transmitter array to the $k$ th receiver array, we denote by $\mathbf{H}_{k}$ the channel matrix from the $k$ th transmitter array to the $k$ th receiver array for simplified notation. Hence, $\mathbf{H}_{k}=\mathbf{H}_{k k}$. In the current paper, we use spectral efficiency and capacity interchangeably, both refer to the mutual information per unit time and unit bandwidth for a given system or user. We reserve throughput to denote the bits per second for a given system or user with a given bandwidth. This distinction is important in Section V where we discuss the limiting capacity of C-MIMO.

\section{NETwork Model AND Assumptions}

Consider an ad hoc network with $K$ simultaneously communicating transmitter-receiver pairs. Each transmitter is equipped with $t$ transmit antennas and receiver with $r$ receive antennas. A simple approach to accommodate $K$ transceiver pairs is to divide the available time-frequency into orthogonal channels through time-division multiple-access (TDMA) or 
frequency-division multiple-access (FDMA) or combinations thereof. Each transmitter-receiver pair will then conduct single-user MIMO communication in each subchannel without interference.

In this work, we investigate the performance gain, if any, of using the inherent multiplexing gain in MIMO communication to accommodate multiuser communication. The system layout is essentially the same as that of Blum [12] where all MIMO nodes communicate in the same channel and each transceiver pair attempts to do interference suppression through the use of multiple receiver antennas. Thus, we are shifting some of the high-layer function (i.e., medium access control) into the physical layer.

The following assumptions are used in the ensuing derivations.

A1) All users have identical power constraint $P$ unless otherwise stated.

A2) We assume a rich scattering environment: each channel matrix consists of independent identically distributed (i.i.d.) complex Gaussian random variables with zero mean and unit variance; i.e., real and imaginary parts are $\mathcal{N}(0,1 / 2)$. Notice that this rich scattering assumption may eventually break down as the number of antennas becomes large [17].

A3) The combined path loss/shadow fading, denoted by $\eta_{k j}$ for the channel between the $j$ th transmitter and the $k$ th receiver is i.i.d. with mean $\bar{\eta}$. Further $\eta_{k j}$ (large scale fading) is independent of the channel matrix $\mathbf{H}_{k j}$ which captures small scale fading.

A4) Circularly complex Gaussian noise with covariance matrix $\sigma^{2} \mathbf{I}_{r}$ is assumed at each receiver, where $\sigma^{2}$ is the noise power over the whole channel bandwidth.

A5) Gaussian codes are assumed for each user. This does not lose any optimality in the presence of additive Gaussian noise.

A6) The CSI corresponding to the desired transmitter-receiver pair is available at the receiver. In Section IV, it is also assumed that such CSI is also available at the transmitter.

We remark here that assumption A1 has a very significant impact on the limiting capacity for C-MIMO. Fixing per-user transmit power results in an unbounded total power when more users are added to the system, which in turn gives C-MIMO an unbounded spectral efficiency as shown in Section V.

Assume that the transmit vector for the $j$ th transmitter has a covariance matrix $\mathbf{R}_{j}$, the ergodic mutual information (MI) for the $k$ th transmitter-receiver pair is

$$
\begin{aligned}
C_{k}=\mathcal{E}\left[\log \mid \mathbf{I}_{r}\right. & +\eta_{k} \mathbf{H}_{k} \mathbf{R}_{k} \mathbf{H}_{k}^{H} \\
& \left.\times\left(\sigma^{2} \mathbf{I}_{r}+\sum_{j \neq k} \eta_{k j} \mathbf{H}_{k j} \mathbf{R}_{j} \mathbf{H}_{k j}^{H}\right)^{-1} \mid\right] .
\end{aligned}
$$

The ergodic expectation $\mathcal{E}$ averages over all instantiations of $\left\{\eta_{k}\right\}$ and $\left\{\mathbf{H}_{k j}\right\}$. Notice that in the cases where the transmitter knows CSI, therefore $\mathbf{R}_{k}$ is a function of CSI, the above definition implies a block fading channel model where each fading state remains stationary long enough so that the corresponding deterministic MI corresponding to the given channel instantiation can be achieved. The distinction between the deterministic and ergodic capacities for fading channels is articulated in [18].

The network spectral efficiency is defined as the sum of pairwise spectral efficiencies between intended transmitter-receiver pairs; i.e.,

$$
C=\sum_{k=1}^{K} C_{k} .
$$

To facilitate our ensuing development, we first introduce the following lemma.

Lemma 1: If $\mathbf{H}$ is a $r \times t$ matrix with i.i.d. zero mean unit variance entries, $\mathbf{R}$ is a $t \times t$ Hermitian and positive semidefinite matrix with trace $a$, then

$$
\mathcal{E}\left[\mathbf{H R H}^{H}\right]=a \mathbf{I} .
$$

Proof: The eigen decomposition of $\mathbf{R}$ yields

$$
\mathbf{R}=\mathbf{U} \mathbf{\Lambda} \mathbf{U}^{H}
$$

where $\mathbf{U}$ is a unitary matrix and $\Lambda$ is a diagonal matrix with $\sum_{i} \lambda_{i}=a$. Thus

$$
\mathbf{H R H} \mathbf{H}^{H}=\mathbf{H} \mathbf{U} \boldsymbol{\Lambda} \mathbf{U}^{H} \mathbf{H}^{H}=\tilde{\mathbf{H}} \boldsymbol{\Lambda} \tilde{\mathbf{H}}^{H}
$$

where $\tilde{\mathbf{H}}=\mathbf{H U}$. Given that $\mathbf{U}$ is unitary, $\tilde{\mathbf{H}}$ and $\mathbf{H}$ have identical first- and second-order moments. Now

$$
\begin{aligned}
\mathcal{E}\left[\mathbf{H R H}^{H}\right] & =\mathcal{E}\left[\tilde{\mathbf{H}} \boldsymbol{\Lambda} \tilde{\mathbf{H}}^{H}\right]=\mathcal{E}\left[\sum_{i=1}^{t} \lambda_{i} \tilde{\mathbf{h}}_{i} \tilde{\mathbf{h}}_{i}^{H}\right] \\
& =\sum_{i=1}^{t} \lambda_{i} \mathcal{E}\left[\tilde{\mathbf{h}}_{i} \tilde{\mathbf{h}}_{i}^{H}\right]=\left(\sum_{i=1}^{t} \lambda_{i}\right) \mathbf{I}=a \mathbf{I} .
\end{aligned}
$$

While Lemma 1 holds for a deterministic matrix $\mathbf{R}$, it can be trivially extended to cases where $\mathbf{R}$ is a Hermitian and positive semidefinite random matrix with the same trace constraint, as long as it is independent of $\mathbf{H}$.

\section{Network SPECTRAL EFFICIENCY IN THE AbSENCE OF CSI}

In [12], it was shown that in the absence of CSI at the transmitter in MIMO ad hoc networks, the optimal signaling depends on the interference to noise power ratio. In particular, with weak interference, the transmitter should put equal power on all antennas (i.e., using the optimal interference free transmission [3], termed herein as the "weak interference" mode); while with strong interference, the transmitter should operate in a "singular" mode: it puts all its power on a single antenna (which is equivalent to transmitting identical information through all antennas). We establish in this section that with both channel blind transmission schemes, the asymptotic spectral efficiency is limited solely by the receive antenna size $r$. As such, to achieve meaningful per-user spectral efficiency, one needs to scale up $r$ 
to the same order as the number of active transmitter-receiver pairs.

In reference to (1) and (2), the weak interference mode uses $\mathbf{R}=(P / t) \mathbf{I}_{t}$ as the covariance matrix at the transmitter. For the singular mode, without loss of generality, we assume that each transmitter puts all the power on its first antenna element. Consequently, the covariance matrix is of the form, for all transmitters

$$
\mathbf{R}=\left[\begin{array}{cccc}
P & 0 & \cdots & 0 \\
0 & 0 & \cdots & 0 \\
\vdots & \vdots & \ddots & \vdots \\
0 & 0 & \cdots & 0
\end{array}\right]
$$

In both cases, we have

$$
\operatorname{trace}(\mathbf{R})=P
$$

To formally establish the limiting spectral efficiency, recall from (1) and (2), and using the fact that $\mathbf{R}_{k}$ is identical for all $k$, we have

$$
\begin{aligned}
C=\sum_{k=1}^{K} \mathcal{E}\left[\log \mid \mathbf{I}_{r}+\right. & \eta_{k} \mathbf{H}_{k} \mathbf{R} \mathbf{H}_{k}^{H} \\
& \left.\times\left(\sigma^{2} \mathbf{I}_{r}+\sum_{j \neq k} \eta_{k j} \mathbf{H}_{k j} \mathbf{R} \mathbf{H}_{k j}^{H}\right)^{-1} \mid\right] .
\end{aligned}
$$

Let $K \rightarrow \infty$ and we have, by the law of large number (LLN)

$$
\begin{aligned}
\lim _{K \rightarrow \infty} \frac{1}{K-1} \sum_{j \neq k} \eta_{k j} \mathbf{H}_{k j} \mathbf{R H}_{k j}^{H} & =\mathcal{E}\left[\eta_{k j} \mathbf{H}_{k j} \mathbf{R} \mathbf{H}_{k j}^{H}\right] \\
& =\bar{\eta} \mathcal{E}\left[\mathbf{H}_{k j} \mathbf{R} \mathbf{H}_{k j}^{H}\right] .
\end{aligned}
$$

From Lemma 1, we have, for both singular and the weak interference modes

$$
\mathcal{E}\left[\mathbf{H}_{k j} \mathbf{R H}_{k j}^{H}\right]=P \mathbf{I}_{r}
$$

Therefore

$$
\begin{aligned}
C \stackrel{\rightarrow^{\infty}}{\mathcal{E}}\left[\sum_{k=1}^{K} \log \mid \mathbf{I}_{r}+\eta_{k} \mathbf{H}_{k} \mathbf{R} \mathbf{H}_{k}^{H}\right. \\
\left.\quad \times\left(\sigma^{2} \mathbf{I}_{r}+P(K-1) \bar{\eta} \mathbf{I}_{r}\right)^{-1} \mid\right] \\
=\sum_{k=1}^{K} \mathcal{E}\left[\log \left|\mathbf{I}_{r}+\frac{1}{\sigma^{2}+P \bar{\eta}(K-1)} \eta_{k} \mathbf{H}_{k} \mathbf{R} \mathbf{H}_{k}^{H}\right|\right] \\
\leq \sum_{k=1}^{K} \log \left|\mathcal{E}\left[\mathbf{I}_{r}+\frac{1}{\sigma^{2}+P \bar{\eta}(K-1)} \eta_{k} \mathbf{H}_{k} \mathbf{R} \mathbf{H}_{k}^{H}\right]\right| \\
=\sum_{k=1}^{K} \log \left|\mathbf{I}_{r}+\frac{\bar{\eta} P}{\sigma^{2}+P \bar{\eta}(K-1)} \mathbf{I}_{r}\right| \\
=r K \log \left(1+\frac{\bar{\eta} P}{\sigma^{2}+P \bar{\eta}(K-1)}\right) \\
K \rightarrow \infty
\end{aligned}
$$

where the inequality follows from Jensen's inequality and the concavity of the $\log |\cdot|$ function [19] and (4) follows from the fact that

$$
\lim _{x \rightarrow 0} \frac{\log (1+x)}{x}=1
$$

\section{Remarks:}

- In both cases, the per-user spectral efficiency is only $r / K$ for large $K$. To achieve nonzero per-user spectral efficiency, one needs to scale up $r$ to the same order of $K$.

- The fact that the spectral efficiency is independent of the transmit power is because as $K$ increases, interference dominates receiver noise. Assume equal power constraint, the interfering power and the signal power increase proportionally hence the resulting spectral efficiency is invariant to $P$. This, of course, is a direct result of assuming single-user detection, which gives rise to (1) that treats all unintended users' transmissions as equivalent channel noise.

- The transmit antenna size is irrelevant in both the weak interference mode and the singular mode due to the absence of CSI at the transmitters.

- The assumption of a homogeneous network where both $\mathcal{E}\left[\eta_{k k}\right]=\mathcal{E}\left[\eta_{k j}\right]$ may not be true in practice. In an ad hoc network, each node may be more inclined (or constrained) to communicate to its close neighbors. In that case, a homogenous model for all $\eta_{k j}$ is no longer valid. One simple modification is to assume that $\eta_{k k}$ has a larger expected value, denoted by $\bar{\eta}_{0}$, than that of $\eta_{k j}$ for $k \neq j$, denoted by $\bar{\eta}$. Under this simple model, one can establish that the limiting throughput is bounded by

$$
C \leq r \frac{\bar{\eta}_{0}}{\bar{\eta}}
$$

Thus, a higher spectral efficiency may be achieved due to the improved signal-to-interference power ratio (SIR).

- The derivation assumes a static channel model as $K$ increases, i.e., the statistics of $\eta_{k j}$ do not change as $K$ increases. This may not be true-as $K$ increases, the node density increases, hence one may expect $\eta_{k j}$ to become large. Nonetheless, if the expected values of both $\eta_{k k}$ and $\eta_{k j}$ scale with the same order of $K$, the final capacity result still stands.

- Equation (3) deserves some further attention. The pre-log term, $r K$, typically referred to as the "rate" of a MIMO system, seems to indicate a very promising capacity result for MIMO interference transmission-it scales linearly in $K$. However, for large $K$, each MIMO link is effectively operating at the low-signal-to-noise ratio (SNR) regime. This also renders the log term linear, resulting in the loss of $K$ factor in the final limiting capacity.

However, (3) may suggest that with proper care, interference transmission may actually enjoy a capacity advantage for finite $K$. For example, if the desired transceiver pairs enjoy larger $\eta_{k k}$ compared with $\eta_{k j}$, which renders the overall SIR relatively large, the pre-log term may dominate the logarithmic decaying of the SIR, which gives rise 
to a better spectral efficiency for finite $K$. More detailed study, however, is beyond the scope of the present paper.

\section{Network Spectral EfFiciency With CSI AT THE TRANSMITTER}

Consider the $k$ th transmitter-receiver pair whose channel matrix is $\mathbf{H}_{k}$. Without this information at the transmitter, the transmitter either transmits an i.i.d. vector with $\mathbf{R}=(P / t) \mathbf{I}_{t}$ or puts all power on a single antenna (the singular mode). In both cases, the absence of CSI does not allow the transmitter to choose any favorable subspace (eigenmodes) for interference suppression/avoidance.

With $\mathbf{H}_{k}$ available at the $k$ th transmitter, it is reasonable to expect that better spectral efficiency may result. In particular, since the transmitter can utilize its multiple antennas for interference suppression/avoidance, one expects that the achievable spectral efficiency also depends on the number of transmit antennas. We show in the following that this is indeed the case. By limiting the transmitter processing to simple beamforming, we obtain an asymptotic spectral efficiency of $t+r+2 \sqrt{t r}$ which scales both in $t$ and in $r$.

Consider, for the $k$ th user, one uses a beamforming vector $\sqrt{P} \mathbf{c}_{k}$, with $\left\|\mathbf{c}_{k}\right\|=1$ and $\mathbf{c}_{k}$ is determined solely using the channel matrix $\mathbf{H}_{k}$; i.e., $\mathbf{c}_{k}=\mathbf{c}_{k}\left(\mathbf{H}_{k}\right)$. The network spectral efficiency, assuming $K$ total transmitter-receiver pairs, is now

$$
\begin{aligned}
I=\sum_{k=1}^{K} \mathcal{E}\left[\log \mid \mathbf{I}_{r}+\eta_{k} P \mathbf{H}_{k} \mathbf{c}_{k} \mathbf{c}_{k}^{H} \mathbf{H}_{k}^{H}\right. \\
\left.\times\left(\sigma^{2} \mathbf{I}_{r} \sum_{j \neq k} \eta_{k j} P \mathbf{H}_{k j} \mathbf{c}_{j} \mathbf{c}_{j}^{H} \mathbf{H}_{k j}^{H}\right)^{-1} \mid\right] \\
\underset{k \rightarrow \infty}{K} \sum_{k=\mathbf{I}}^{K} \mathcal{E}\left[\log \mid \mathbf{I}_{r}+\eta_{k} P \mathbf{H}_{k} \mathbf{c}_{k} \mathbf{c}_{k}^{H} \mathbf{H}_{k}^{H}\right. \\
\left.\quad \times\left(\sigma^{2} \mathbf{I}_{r}+P(K-1) \bar{\eta} \mathbf{I}_{r}\right)^{-1} \mid\right] \\
=\sum_{k=\mathbf{I}}^{K} \mathcal{E}\left[\log \left|\mathbf{I}_{r}+\frac{\eta_{k} P}{\sigma^{2}+P \bar{\eta}(K-1)} \mathbf{H}_{k} \mathbf{c}_{k} \mathbf{c}_{k}^{H} \mathbf{H}_{k}^{H}\right|\right]
\end{aligned}
$$

where we again use Lemma 1 in the second equality because of the fact that $\mathbf{c}_{k}$ is determined by $\mathbf{H}_{k}$, and hence is independent of $\mathbf{H}_{k j}$ for $j \neq k$. We comment here that the singular mode described in Section III is a special case of the beamforming scheme with $\mathbf{c}_{k}=[1,0, \cdots, 0]^{T}$. With knowledge of $\mathbf{H}_{k}$ at the transmitter, one naturally expects to find a better $\mathbf{c}_{k}$ that maximizes the mutual information. Using $\log |\mathbf{I}+\mathbf{A B}|=$ $\log |\mathbf{I}+\mathbf{B A}|$, we have

$$
\begin{aligned}
\log \mid \mathbf{I}_{r}+ & \frac{\eta_{k} P}{\sigma^{2}+P \bar{\eta}(K-1)} \mathbf{H}_{k} \mathbf{c}_{k} \mathbf{c}_{k}^{H} \mathbf{H}_{k}^{H} \mid \\
& =\log \left(1+\frac{\eta_{k} P}{\sigma^{2}+P \bar{\eta}(K-1)} \mathbf{c}_{k}^{H} \mathbf{H}_{k}^{H} \mathbf{H}_{k} \mathbf{c}_{k}\right) .
\end{aligned}
$$

Clearly, maximizing the quadratic term inside the logarithm in (5) subject to a norm constraint (i.e., $\left\|\mathbf{c}_{k}\right\|=1$ ) yields a beamforming vector $\mathbf{c}_{k}$ that coincides with the eigenvector corresponding to the largest eigenvalue of the matrix $\mathbf{H}_{k}^{H} \mathbf{H}_{k}$, which we denote by $\mathbf{v}_{1}$. We now try to quantify the network spectral efficiency of this simple beamforming approach. First, $\mathbf{c}_{k}^{H} \mathbf{H}_{k}^{H} \mathbf{H}_{k} \mathbf{c}_{k}=\mathbf{v}_{1}^{H} \mathbf{H}_{k}^{H} \mathbf{H}_{k} \mathbf{v}_{1}=\lambda_{1}^{(k)}$ where $\lambda_{1}^{(k)}$ is the maximum eigenvalue of the matrix $\mathbf{H}_{k}^{H} \mathbf{H}_{k}$. To find the corresponding mutual information, we have

$$
\begin{aligned}
I & =\sum_{k=1}^{K} \mathcal{E}\left[\log \left(1+\frac{\eta_{k} \lambda_{1}^{(k)} P}{\sigma^{2}+P \bar{\eta}(K-1)}\right)\right] \\
& \leq \sum_{k=1}^{K} \log \mathcal{E}\left[1+\frac{\eta_{k} \lambda_{1}^{(k)} P}{\sigma^{2}+P \bar{\eta}(K-1)}\right] \\
& =\sum_{k=1}^{K} \log \left[1+\frac{\bar{\eta} E\left[\lambda_{1}^{(k)}\right] P}{\sigma^{2}+P \bar{\eta}(K-1)}\right] \\
& \stackrel{\rightarrow}{\rightarrow} E\left[\lambda_{1}^{(k)}\right]
\end{aligned}
$$

where we used Jensen's inequality for the concave function $\log (\cdot)$. To compute $E\left[\lambda_{1}^{(k)}\right]$, we notice that $\mathbf{H}_{k}$ being a channel matrix of complex Gaussian i.i.d. entries, $\mathbf{H}_{k}^{H} \mathbf{H}_{k}$ is essentially a sample covariance matrix of a vector random variable $\mathbf{h} \sim$ $\mathcal{C N}(0, \mathbf{I})$. From [20] and [21], we know that for large $t, r^{1}$

$$
\mathcal{E}\left[\lambda_{1}^{(k)}\right] \approx(\sqrt{t}+\sqrt{r})^{2} .
$$

Thus, the total spectral efficiency is now bounded by

$$
I \leq t+r+2 \sqrt{t r} .
$$

This upper bound is indeed achievable asymptotically. To show this, from (6) and letting $K \rightarrow \infty$, one has, using the fact that $\log (1+x) \approx x$ for small $x$

$$
\log \left(1+\frac{\eta_{k} \lambda_{1}^{(k)} P}{\sigma^{2}+P \bar{\eta}(K-1)}\right) \approx \frac{\eta_{k} \lambda_{1}^{(k)} P}{\sigma^{2}+P \bar{\eta}(K-1)} .
$$

Taking expectation with respect to $\lambda_{1}^{(k)}$ and $\eta_{k}$ and then sum over $k$ yields

$$
I=\mathcal{E}\left[\lambda_{1}^{(k)}\right] \approx t+r+2 \sqrt{t r} .
$$

Since this result is derived by restricting to a beamforming approach, the actual achievable spectral efficiency, i.e., the capacity, is lowered bounded by $t+r+2 \sqrt{t r}$, i.e.,

$$
C>t+r+2 \sqrt{t r}
$$

for large $t$ and $r$.

The above derivation assumes that the transmitter has exact knowledge of CSI. With imperfect CSI, where the beamforming vector $\mathbf{c}_{k}$ is misaligned with the eigenvector corresponding to the largest eigenvalue of $\mathbf{H}_{k}^{H} \mathbf{H}_{k}$, we can derive in a similar fashion that the resulting limiting spectral efficiency with the beamforming approach is now

$$
\mathcal{E}\left[\mathbf{c}_{k}^{H} \mathbf{H}_{k}^{H} \mathbf{H}_{k} \mathbf{c}_{k}\right] .
$$

${ }^{1}$ Stronger convergence results exist. Indeed, as $t$ and $r$ both increase to infinity with $r / t$ fixed, the largest eigenvalue converges almost surely to $t+r+\sqrt{t r}$. 
The exact degradation due to the imperfect CSI depends on the particular $\mathbf{c}_{k}$ used in the beamforming approach. We note that (8) is actually quite general; for example, the limiting capacity for the singular mode can be obtained by using $\mathbf{c}_{k}=$ $[1,0, \cdots, 0]^{T}$, which yields $r$ after taking the expectation.

While this lower bound is still independent of $P$, the transmit power, one can improve the spectral efficiency by scaling up $t$ or $r$ or both. The fact that transmitting along the singular direction that has the largest SNR (largest eigenvalue of $\mathbf{H}_{k}^{H} \mathbf{H}_{k}$ ) yields the maximum spectral efficiency is not surprising: since the channel matrices are assumed to be independent, the interference power is evenly distributed among all subspaces when $K$ is large. As such, sending information along only the strongest eigenmode can maximize the SIR among all beamforming approaches. To go one step further, if we assume that the $k$ th transmitter has the CSI corresponding not only to its desired receiver, but all other receivers that it interferes with, one may be able to get an even better spectral efficiency with only a finite number of interferers. We will present in Section VI a heuristic interference suppression beamformer and demonstrate its performance improvement using numerical examples. For the remainder of this section, we will generalize the beamforming idea and present the asymptotically optimum transmitting scheme by assuming only the CSI of the desired transmitter-receiver pair.

Assume that the transmit vector for the $k$ th transmitter has a covariance matrix $\mathbf{R}_{k}$ with trace $\left(\mathbf{R}_{k}\right)=P$. The network spectral efficiency is now

$$
\begin{aligned}
C=\sum_{k=1}^{K} \mathcal{E}\left[\log \mid \mathbf{I}_{r}+\right. & \eta_{k} \mathbf{H}_{k} \mathbf{R}_{k} \mathbf{H}_{k}^{H} \\
& \left.\left.\times\left(\sigma^{2} \mathbf{I}_{r}+\sum_{j \neq k} \eta_{k j} \mathbf{H}_{k j} \mathbf{R}_{j} \mathbf{H}_{k j}^{H}\right)^{-1}\right]\right] .
\end{aligned}
$$

Again, invoking the asymptotic assumption $(K \rightarrow \infty)$ and using Lemma 1, we get

$$
\begin{aligned}
C & \stackrel{K}{ }=\sum_{k=1}^{K} \mathcal{E}\left[\log \left|\mathbf{I}_{r}+\frac{\eta_{k}}{\sigma^{2}+P \bar{\eta}(K-1)} \mathbf{H}_{k} \mathbf{R}_{k} \mathbf{H}_{k}^{H}\right|\right] \\
& =\sum_{k=1}^{K} \mathcal{E}\left[\log \left|\mathbf{I}_{t}+\mathbf{R}_{k} \frac{\eta_{k}}{\sigma^{2}+P \bar{\eta}(K-1)} \mathbf{H}_{k}^{H} \mathbf{H}_{k}\right|\right] .
\end{aligned}
$$

Hence, the asymptotically optimum $\mathbf{R}_{k}$ corresponds to simply waterfilling for the combined channel covariance matrix $\left(\eta_{k} / \sigma^{2}+P \bar{\eta}(K-1)\right) \mathbf{H}_{k}^{H} \mathbf{H}_{k}$. In other words, if we define

$$
\hat{\mathbf{H}}_{H}=\left(\frac{\eta_{k}}{\sigma^{2}+P \bar{\eta}(K-1)}\right)^{1 / 2} \mathbf{H}_{k}
$$

then $\mathbf{R}_{k}$ should be chosen through single-user waterfilling corresponding to the channel matrix $\hat{\mathbf{H}}_{k}$ [3]. Notice this is different than simply scaling the waterfilling solution for $\mathbf{H}_{k}$ : the waterfilling level is determined by the inverse of the eigenvalues of $\hat{\mathbf{H}}_{k}^{H} \hat{\mathbf{H}}_{k}$, and hence depends on the scaling factor in a nonlinear fashion.

\section{CHANNELIZED TRANSMisSION FOR MIMO AD HOC NETWORKS}

For comparison purpose, we describe in detail the C-MIMO and its asymptotic spectral efficiency. Consider a system of bandwidth $B \mathrm{~Hz}$, therefore the total throughput is now $B C$ where $C$ is the network spectral efficiency. Assuming FDMA, ${ }^{2}$ we divide the total bandwidth $B \mathrm{~Hz}$ into $K$ subchannels each with $B_{K}=B / K \mathrm{~Hz}$. Because of the fact that noise variance for each subchannel decreases proportional to the bandwidth, each transmitter-receiver pair has a throughput of

$$
\begin{aligned}
C_{k} B_{K} & =B_{K} \mathcal{E}\left[\log \left|\mathbf{I}_{r}+\eta_{k} \frac{P / t}{\sigma^{2} / K} \mathbf{H}_{k} \mathbf{H}_{k}^{H}\right|\right] \\
& =\frac{B}{K} \mathcal{E}\left[\log \left|\mathbf{I}_{r}+\eta_{k} \frac{K P}{\mathcal{N}_{0} B} \mathbf{H}_{k} \mathbf{H}_{k}^{H}\right|\right]
\end{aligned}
$$

where $\sigma^{2} / K=\mathcal{N}_{0} B / K$ is the noise power for each subchannel. The network throughput is

$$
\begin{aligned}
C B & =\sum_{k=1}^{K} \frac{B}{K} \mathcal{E}\left[\log \left|\mathbf{I}_{r}+\eta_{k} \frac{K P}{t \mathcal{N}_{0} B} \mathbf{H}_{k} \mathbf{H}_{k}^{H}\right|\right] \\
& =B \mathcal{E}\left[\log \left|\mathbf{I}_{r}+\eta_{k} \frac{K P}{t \mathcal{N}_{0} B} \mathbf{H}_{k} \mathbf{H}_{k}^{H}\right|\right]
\end{aligned}
$$

given that $\left(\eta_{k}, \mathbf{H}_{k}\right)$ are assumed to be identically distributed for all $k$. Therefore, the network spectral efficiency of a C-MIMO is

$$
C=\mathcal{E}\left[\log \left|\mathbf{I}_{r}+\eta_{k} \frac{K P}{t \mathcal{N}_{0} B} \mathbf{H}_{k} \mathbf{H}_{k}^{H}\right|\right]
$$

which is simply the ergodic capacity of a single-user MIMO channel with CSI only at the receiver. However, an interesting phenomenon with C-MIMO is that its spectrum efficiency increases logarithmically with $K$ and will approach $\infty$ as $K \rightarrow$ $\infty$. This is due to the assumption of fixed transmitter power $P$ and the fact that more users in the system results in a larger total transmit power while the total noise power remains constant. Or, equivalently, as more users are added, each user occupies a narrower bandwidth, resulting in decreased noise power, and hence improved spectral efficiency.

For fairness of comparison, especially when considering those transmission schemes utilizing CSI presented in Section IV, we will also use, in addition to (10)

$$
C=\mathcal{E}\left[\log \left|\mathbf{I}_{r}+\eta_{k} \frac{K}{\sigma^{2}} \mathbf{H}_{k} \mathbf{R}_{k} \mathbf{H}_{k}^{H}\right|\right]
$$

where $\mathbf{R}_{k}$ is the waterfilling covariance matrix with $\operatorname{trace}\left(\mathbf{R}_{k}\right)=P$, i.e., CSI is assumed also known at the transmitter. Compared to (10), this new spectral efficiency exhibits a performance gain over (10) which remains approximately constant for large SNR.

One can of course impose a different power constraint which will have a great impact on the asymptotic spectral efficiency for C-MIMO. For example, one can fix the sum transmit power

\footnotetext{
${ }^{2}$ An identical result can be obtained by using TDMA and assuming an average power constraint instead of peak power constraint. Thus, each user transmits at $K P$ during its transmission slot.
} 
of all users, that is, $K P=P_{0}$ remains constant. Hence, as $K$ increases, per-user power $P=P_{0} / K$ decreases. With such a constraint, it is easy to show that the network capacity for a C-MIMO is invariant to $K$. In particular, with an ideal MAC, one can show that

$$
C=\mathcal{E}\left[\log \left|\mathbf{I}_{r}+\eta_{k} \frac{P_{0}}{\sigma^{2}} \mathbf{H}_{k} \mathbf{H}_{k}^{H}\right|\right]
$$

which remains constant as a function of $K$. On the other hand, fixing the total transmit power will introduce a constant scaling of $\bar{\eta} P_{0} /\left(\sigma^{2}+\bar{\eta} P_{0}\right)$ to the asymptotic spectral efficiency for interference transmission introduced in Sections III and IV. For example, without CSI and with a total power constraint, the asymptotic network spectral efficiency is now upper bounded by

$$
\frac{\bar{\eta} P_{0}}{\sigma^{2}+\bar{\eta} P_{0}} r \text { nats } / \mathrm{s} / \mathrm{Hz}
$$

while the asymptotic spectral efficiency with limited CSI is now

$$
\frac{\bar{\eta} P_{0}}{\sigma^{2}+\bar{\eta} P_{0}}(t+r+2 \sqrt{t r}) \text { nats/s/Hz. }
$$

The total power constraint has important applications in power limited systems, including, for example, sensor networks. These results can be easily obtained following similar derivations in Section III.

We now discuss carefully the practical implications of C-MIMO compared with all the other interference transmission schemes. We first notice that the network spectral efficiency comparison is SNR dependent: C-MIMO improves as SNR increases while all the interference transmission schemes have spectral efficiencies that are asymptotically independent of SNR. Thus, at very high-SNR regime, C-MIMO has obvious performance advantage. From an implementation point of view, for C-MIMO, each user pair is transmitting in an interference-free channel, therefore the physical layer design is rather straightforward; it amounts to designing single-user MIMO systems operating in orthogonal channels. However, it puts extraordinary burden on the MAC layer in order to achieve the desired network spectral efficiency: one needs to adaptively divide the total channel into orthogonal channels depending on the number of active transmitter-receiver pairs. This may be problematic in practice with dynamic user activities. Overdividing the channel to accommodate all potential users may result in gross channel underutilization as, typically, only a fraction of all users may be active. On the other hand, dynamic channel division and allocation (using, for example, channel contention) runs the risk of denial of access in addition to excessive burden on the MAC layer. The latter effect is especially severe because of the ad hoc network structure where individual nodes are not assumed to have global information of user activities.

The interference transmission schemes simplify the medium access control by allowing all users to transmit in a single channel. It, however, requires more complicated physical

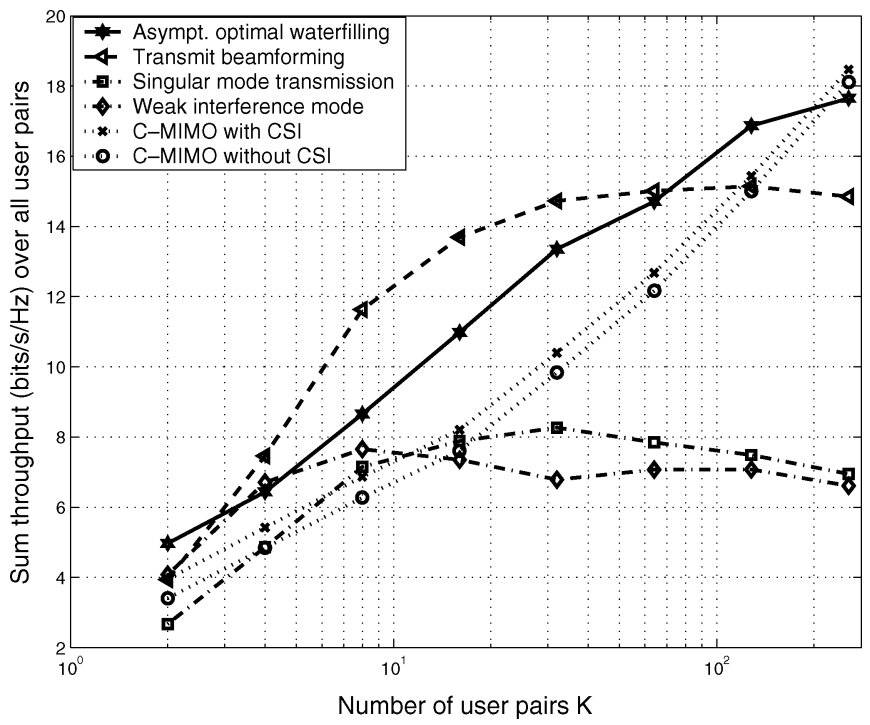

Fig. 1. Sum spectral efficiency of a MIMO ad hoc network with $t=r=4$, $P=2$, and $\sigma^{2}=1$.

layer processing as one needs to deal with interference avoidance/cancellation in order to realize the predicated network spectral efficiency. In addition, since the network spectral efficiency is asymptotically limited by the transceiver antenna numbers, the interference transmission may not be suitable when the nodes of transceiver pairs are exceedingly large compared with the antenna element numbers.

\section{NUMERICAL EXAMPLES}

In this section, we use numerical examples to study the network spectral efficiency of a MIMO ad hoc network with various CSI assumptions and different transmission schemes. In particular, we demonstrate that, with CSI available at the transmitter, substantially larger network spectral efficiency can be achieved than that of the blind transmitter approach. This is in sharp contrast to the single-user MIMO systems where CSI provides an approximately constant yet typically insignificant gain over the blind transmitter in a rich scattering channel environment. The simulation results confirm our theoretical analyses in the preceding sections.

Throughout this section, we assume that the channel matrix from the $j$ th transmitter to the $k$ th receiver, $\mathbf{H}_{k j}$, consists of i.i.d. complex Gaussian entries with zero mean and unit variance. As such, a rich scattering environment with Rayleigh flat fading channel is assumed. The channel matrices are independent across different transmitter-receiver pairs. The path loss/shadowing effect is summarized using the coefficient $\eta_{k j}$ which is assumed to be lognormal distributed, appropriately normalized to have unit mean value (hence the path loss is assumed to be absorbed through appropriately scaling the noise variance). The corresponding standard deviation of average power variation is $10 \mathrm{~dB}$ [22]. Notice that while the variation does not have any impact on the asymptotic spectral efficiency of all the interference transmissions, it does affect the spectral efficiency of C-MIMO, as can be seen from (10) and (11). 


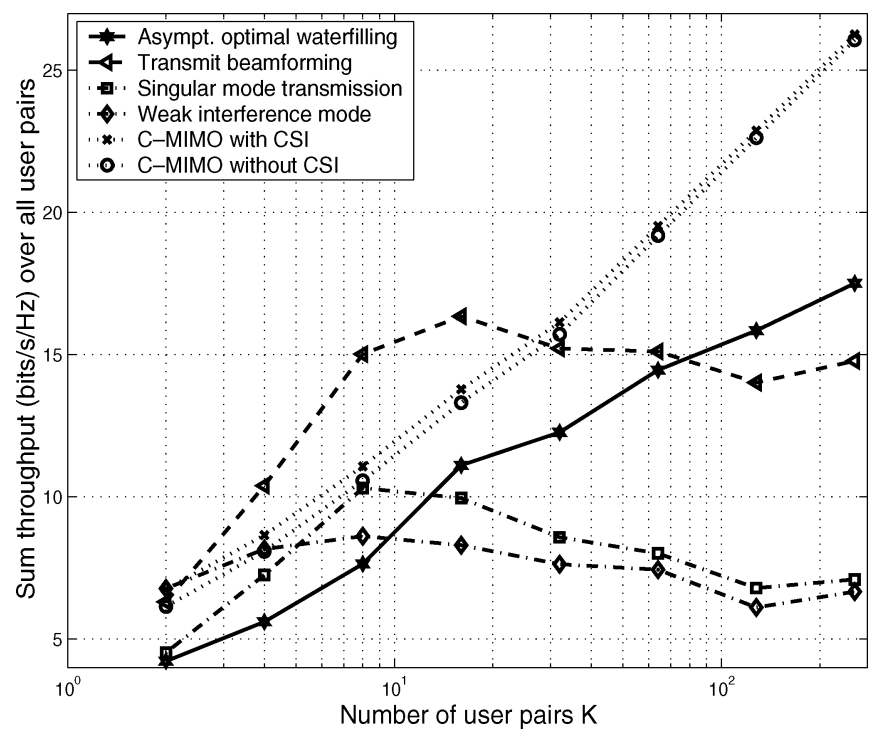

Fig. 2. Sum spectral efficiency of a MIMO ad hoc network with $t=r=4$, $P=10$, and $\sigma^{2}=1$.

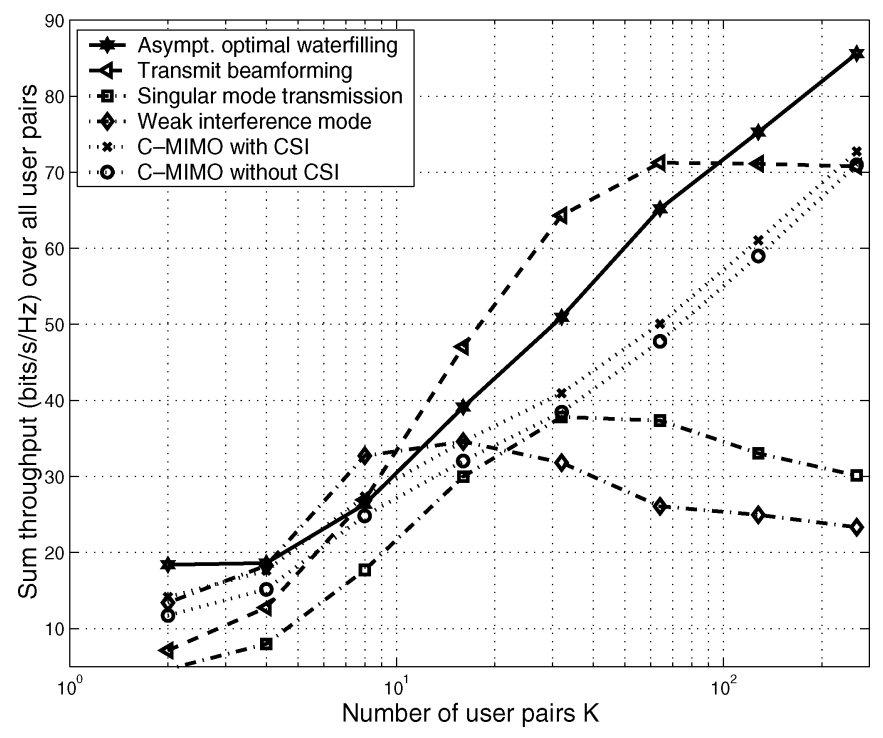

Fig. 3. Sum spectral efficiency of a MIMO ad hoc network with $t=r=16$, $P=2$, and $\sigma^{2}=1$.

Six different transmitting schemes are considered in the following examples:

1) the asymptotically optimal waterfilling approach using the CSI as in (9);

2) simple beamforming approach as described in Section IV;

3) Blum's "singular" transmission mode;

4) Blum's weak interference transmission mode;

5) C-MIMO with CSI, as in (11);

6) C-MIMO without CSI, as in (10).

\section{A. Spectral Efficiency as a Function of $K$}

We plot the sum (network) spectral efficiency as a function of $K$ for different sets of parameters.

1) $t=r=4, P=2, \sigma^{2}=1$. The result is in Fig. 1 .

2) $t=r=4, P=10, \sigma^{2}=1$. The result is in Fig. 2 .

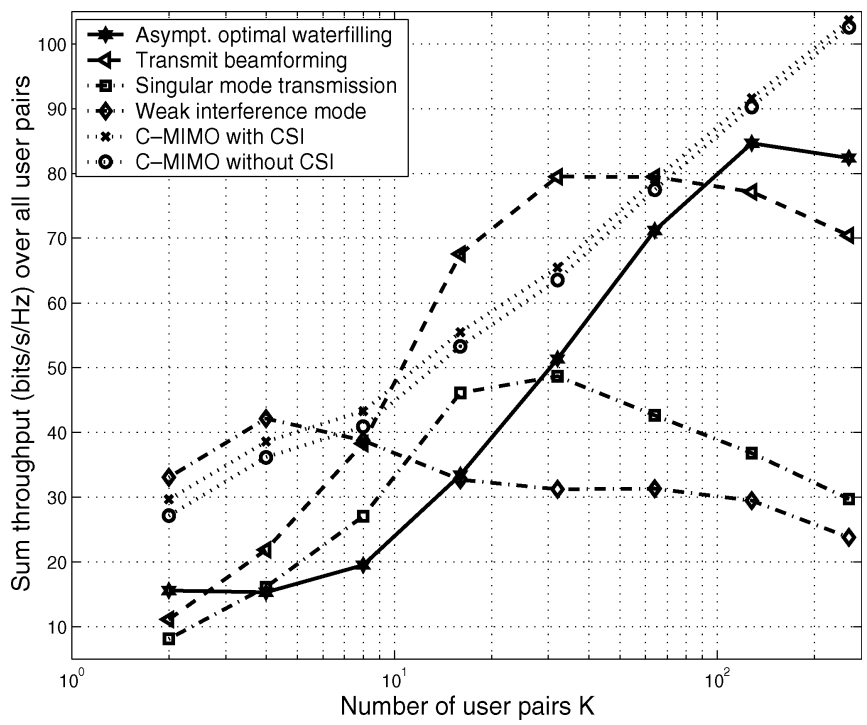

Fig. 4. Sum spectral efficiency of a MIMO ad hoc network with $t=r=16$, $P=10$, and $\sigma^{2}=1$.

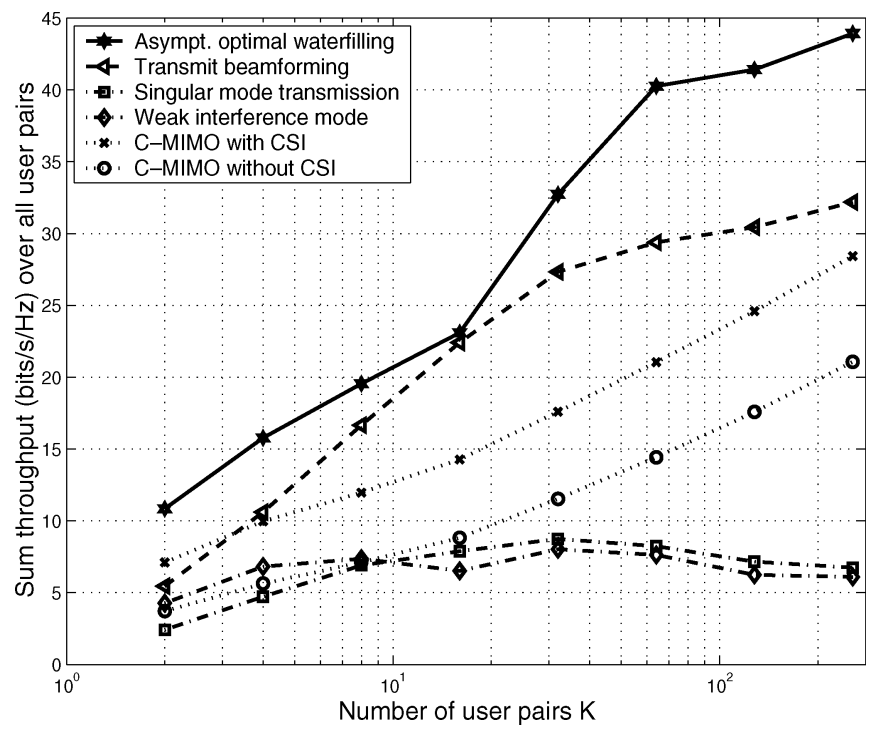

Fig. 5. Sum spectral efficiency of a MIMO ad hoc network with $t=16$, $r=4, P=10$, and $\sigma^{2}=1$.

3) $t=r=16, P=2, \sigma^{2}=1$. The result is in Fig. 3 .

4) $t=r=16, P=10, \sigma^{2}=1$. The result is in Fig. 4 .

5) $t=16, r=4, P=2, \sigma^{2}=1$. The result is in Fig. 5 .

We simulate the ergodic capacity by averaging, for each case, over 50 sets of independently generated channel matrices and shadowing coefficients for all transmitter-receiver pairs.

Remarks:

- As expected, the spectral efficiency for the C-MIMO grows logarithmically as a function of $K$ (linear in the figures as $K$ is plotted in logarithmic scale). This is again based on ideal MAC and with a constant per-user power constraint.

- In all cases, knowing the CSI at the transmitter (the waterfilling and beamforming approaches) improves substantially the network spectral efficiency over the channel-blind transmission schemes (both the weak interference and the singular modes). 


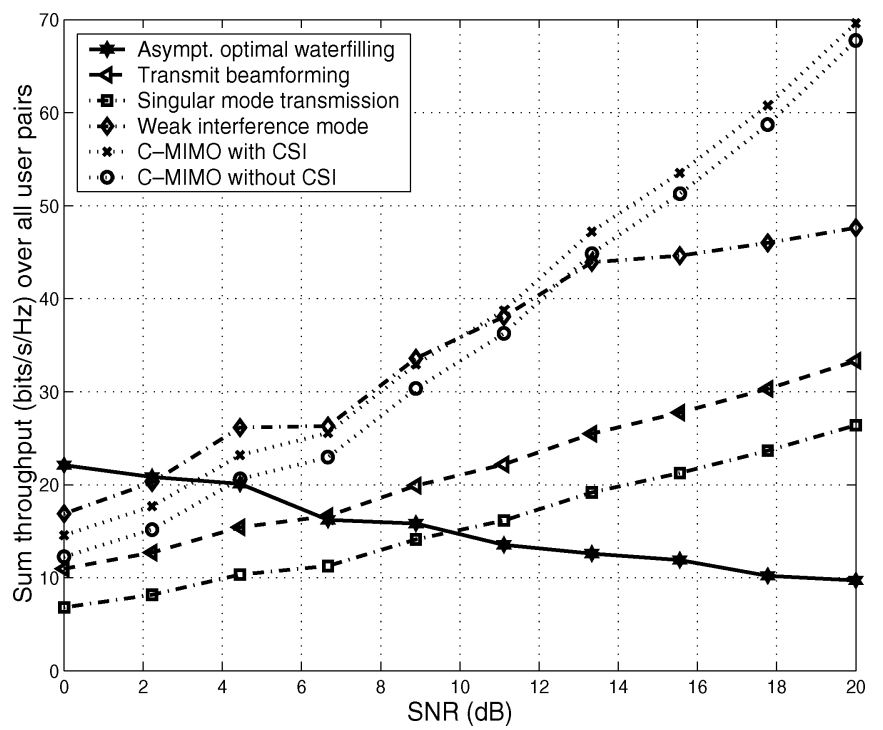

Fig. 6. Sum spectral efficiency of a MIMO ad hoc network with $t=r=16$ and $K=4$.

- $\quad$ Both channel-blind transmission schemes have asymptotic ( $K$ large) spectral efficiency that is close to $r$ nats $/ \mathrm{s} / \mathrm{Hz}$ (or $r \log _{2} e \mathrm{~b} / \mathrm{s} / \mathrm{Hz}$ ). Further, this asymptotic value is independent of the transmit power (compare Figs. 3 and 4) and the transmit antenna size (compare Figs. 2 and 5).

- The asymptotic spectral efficiency for the simple beamforming approach is less than $t+r+2 \sqrt{t r}$. For example, with $t=r=4, t+r+2 \sqrt{t r}=16$ nats/s/Hz, or equivalently, 23 b/s/Hz. From Figs. 1 and 2, the asymptotic spectral efficiency is only $14 \mathrm{~b} / \mathrm{s} / \mathrm{Hz}$. This is because of the fact that (7) is only true asymptotically in $t$ and $r$ (i.e., $r$ and $t$ both are sufficiently large). Otherwise, the distribution of the largest eigenvalue of a sample covariance matrix is skewed toward smaller values and thus a smaller expected value results. Increasing $t$ and $r$ will improve the accuracy of this approximation. For example, for $t=r=16$, the predicted spectral efficiency using (7) is $92 \mathrm{~b} / \mathrm{s} / \mathrm{Hz}$ while the actual spectral efficiency from Figs. 3 and 4 is $70 \mathrm{~b} / \mathrm{s} / \mathrm{Hz}$ which is a much better approximation compared to the case of $t=r=4$.

- As evidenced in all cases, as the number of users $K$ becomes very large, the asymptotically optimum waterfilling approach will outperform the simple beamforming approach.

\section{B. Spectral Efficiency as a Function of SNR}

We now plot the network spectral efficiency as a function of SNR for a given number of users. In particular, we plot the following.

1) $t=r=16, K=4, \sigma^{2}=1, P$ varies from 1 to 10 (corresponding SNR ranges from 0 to $20 \mathrm{~dB}$ ). The result is in Fig. 6.

2) $t=r=16, K=16, \sigma^{2}=1, P$ varies from 1 to 10 (corresponding SNR ranges from 0 to $20 \mathrm{~dB}$ ). The result is in Fig. 7.

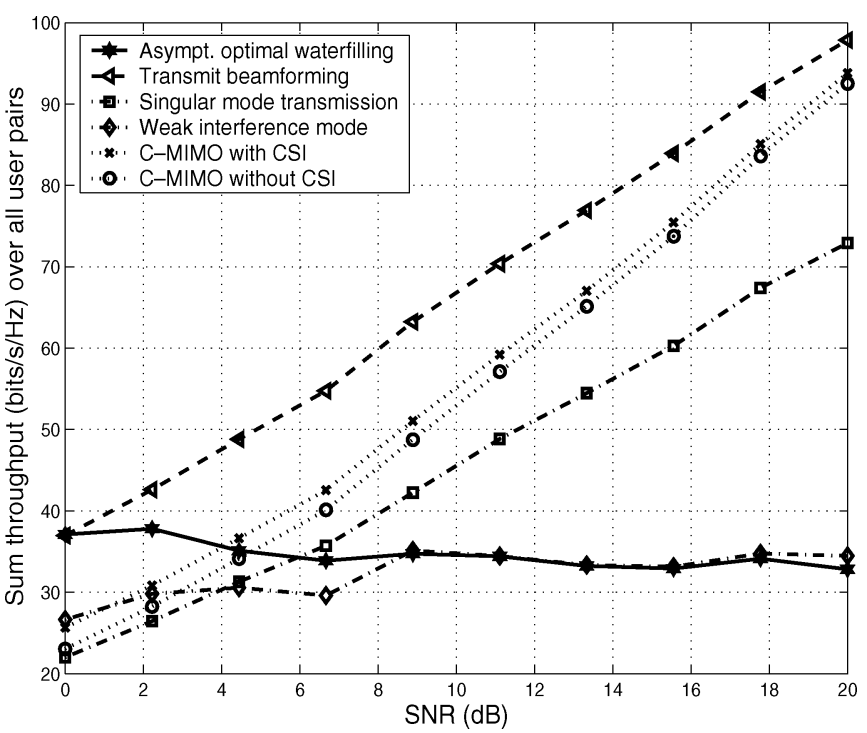

Fig. 7. Sum spectral efficiency of a MIMO ad hoc network with $t=r=16$ and $K=16$.

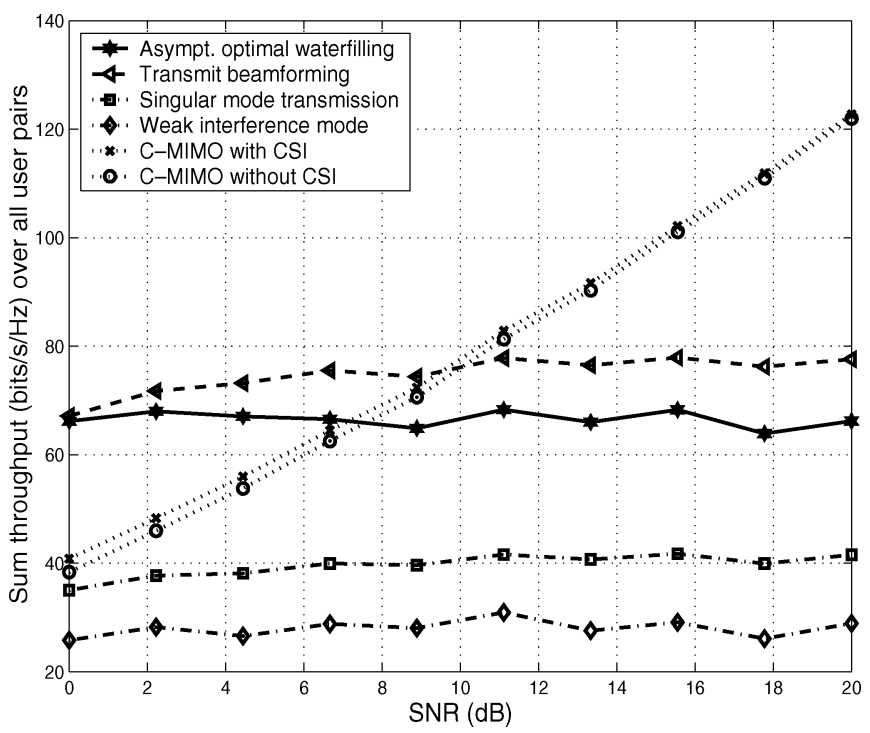

Fig. 8. Sum spectral efficiency of a MIMO ad hoc network with $t=r=16$ and $K=64$.

3) $t=r=16, K=64, \sigma^{2}=1, P$ varies from 1 to 10 (corresponding SNR ranges from 0 to $20 \mathrm{~dB}$ ). The result is in Fig. 8.

\section{Remarks:}

- $\quad$ Figs. 6-8 are consistent with that of Figs. 3 and 4. For example, at SNR $=10 \mathrm{~dB}$ and with $K=64$ transceiver pairs, both Figs. 8 and 4 suggest a network spectral efficiency of approximately $68 \mathrm{~b} / \mathrm{s} / \mathrm{Hz}$ for the waterfilling approach.

- Depending on the number of users and the SNR ranges, interference transmission may actually enjoy a capacity advantage even compared with C-MIMO using an ideal MAC. For example, the simple beamforming approach has comparable or better spectral efficiency performance for moderate value of $K$ for the SNR range under consideration 
(see Fig. 7). This is because interference transmission allows the system to exploit multiuser diversity due to the independent channel assumption. As $K$ increases from 4 to 16 so does the multiuser diversity which results in improved spectral efficiency. When $K$ becomes very large ( $K=64$ as in Fig. 8), the spectral efficiency manifests its limiting behavior, as per the asymptotic analysis.

- Not all the schemes have improved spectral efficiency as SNR increases. This is not surprising-due to the equal transmit power constraint, for any given user, increasing SNR also implies increased interference power as the interfering users' transmit power also increases. The only exception is the C-MIMO schemes where interference-free transmission is assumed; therefore, its capacity is monotone increasing as SNR improves in all cases.

- The asymptotic optimal waterfilling relies on the assumption that the sum interference reduces to white Gaussian vector with large $K$. Thus, it is only optimal when $K$ becomes very large. This can be observed from Figs. 1-5 where the waterfilling approach will eventually outperform the beamforming approach when $K$ grows very large. For finite $K$, however, there is no guarantee that the waterfilling approach performs better than the single beamforming approach. Intuitively, waterfilling approach spreads transmit power along all eigenmodes of the channel matrix, leaving the transmission more liable to strong interference for finite $K$ for which the interference is nonwhite. Beamforming, on the other hand, chooses a single direction to transmit. As channel matrices are assumed uncorrelated, it is less likely that the beamforming direction may coincide with other strong interference.

\section{Interference Suppression Beamforming}

Finally, we show that better spectral efficiency may result with stronger CSI assumption. Consider now each transmitter has the knowledge of not only CSI corresponding to its desired receiver, but to all the other receivers it interferes with. That is, the $k$ th transmitter knows the channel matrices $\mathbf{H}_{i k}$ for $i=1, \cdots, K$. Consider the following heuristic design criterion:

$$
\begin{array}{ll}
\max _{\mathbf{c}_{k}} & \frac{\mathbf{c}_{k}^{H} \mathbf{H}_{k}^{H} \mathbf{H}_{k} \mathbf{c}_{k}}{\mathbf{c}_{k}^{H}\left(\sum_{i \neq k} \mathbf{H}_{i k}^{H} \mathbf{H}_{i k}\right) \mathbf{c}_{k}} \\
\text { s.t. } & \mathbf{c}_{k}^{H} \mathbf{c}_{k}=P
\end{array}
$$

i.e., one wants to maximize the ratio between the signal power at the $k$ th receiver and the interference power from the $k$ th transmitter to all other receivers. ${ }^{3}$ The solution to this maximization problem is easily solved using the generalized eigendecomposition [23]; i.e., $\mathbf{c}_{k}$ should be the eigenvector corresponding to the largest eigenvalue for the following generalized eigenvalue problem, subject to the power constraint

$$
\mathbf{H}_{k}^{H} \mathbf{H}_{k} \mathbf{c}=\lambda\left(\sum_{i \neq k} \mathbf{H}_{i k}^{H} \mathbf{H}_{i k}\right) \mathbf{c} .
$$

\footnotetext{
${ }^{3}$ Note this is not the typical signal to interference power ratio corresponding to a given receiver.
}

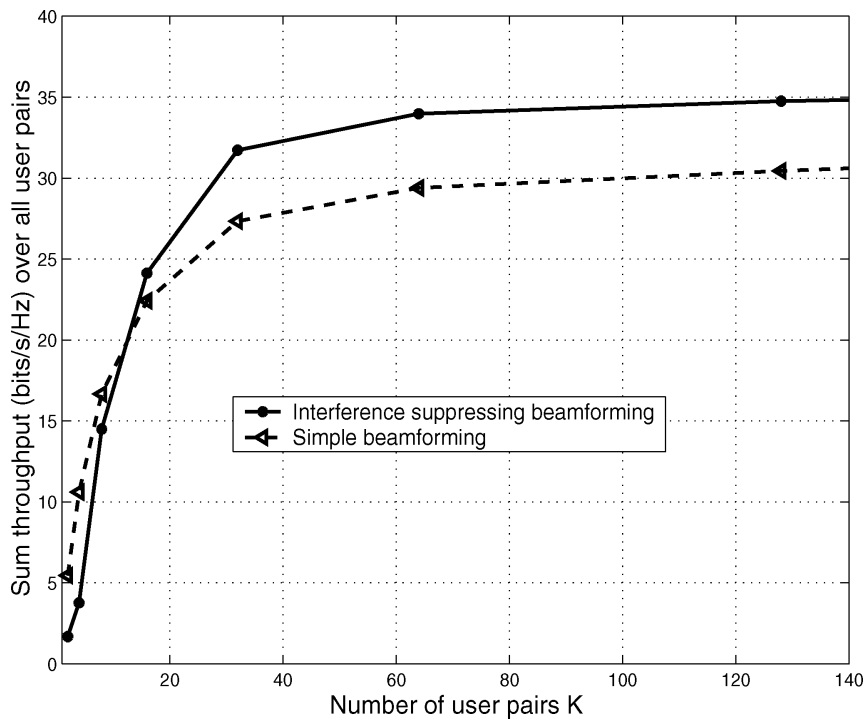

Fig. 9. Spectral efficiency comparison between the simple beamforming and the interference suppressing beamforming for a MIMO ad hoc network with $t=16, r=4, P=10$, and $\sigma^{2}=1$.

Fig. 9 is a comparison of the simple beamforming and the interference suppressing beamforming. As $K$ grows, the interference suppression beamforming approach provides a steady improvement over the simple beamforming approach.

\section{CONCLUSION}

MIMO communications in an ad hoc network is studied in this paper. Communicating in an interference rich environment, we demonstrated that the knowledge of CSI at the transmitter is instrumental in obtaining higher network spectral efficiency. In particular, we show that without CSI at the transmitter, the network spectral efficiency is fundamentally limited by the receiver antenna element: the overall asymptotic spectral efficiency is bounded by $r$ nats $/ \mathrm{s} / \mathrm{Hz}$, where $r$ is the number of antennas at each receiver. With CSI available at the transmitter, the spectral efficiency is approximately $t+r+2 \sqrt{t r}$ nats/s/ $/ \mathrm{Hz}$ for large $t$ and $r$ with a simple beamforming approach. Further, we demonstrated using numerical examples that with a stronger CSI assumption, potentially higher spectral efficiency may result.

The incentive of using "interference transmission" in MIMO ad hoc networks is to simplify the medium access control task, which becomes difficult due to the lack of a base station. This is at the cost of complicating physical layer processing as interference suppression needs to be incorporated in the physical layer design. In essence, utilizing the multiplexing capability of MIMO transceivers provides a meaningful way for cross-layer tradeoff. This motivates further research in cross-layer design for MIMO ad hoc networks that leverage the inherent multiplexing gain in each MIMO transceiver. Moreover, certain applications may warrant more advanced transceiver design, such as superposition code transceivers [14]. Combined with MIMO transceivers, these advanced transceiver structures may have potentially significant impact on the overall network throughput. 


\section{REFERENCES}

[1] G. J. Foschini, "Layered space-time architecture for wireless communication in a fading environment when using multielement antennas," Bell Labs Tech. J., vol. 1, pp. 41-59, Autumn 1985.

[2] G. J. Foschini and M. J. Gans, "On limits of wireless communications in a fading environment when using multiple antennas," Wireless Personal Commun., vol. 6, pp. 311-335, 1998.

[3] E. Telatar, "Capacity of multi-antenna Gaussian channels," Eur. Trans. Telecomm., vol. 10, pp. 585-595, Nov./Dec. 1999.

[4] G. Caire and S. Shamai, "On the achievable throughput of a multiantenna Gaussian broadcast channel," IEEE Trans. Inf. Theory, vol. 49, pp. 1691-1706, Jul. 2003.

[5] S. Vishwanath, S. Jafar, and A. Goldsmith, "Duality, achievable rates, and sum-rate capacity of Gaussian MIMO broadcast channels," IEEE Trans. Inf. Theory, vol. 49, pp. 2658-2668, Oct. 2003.

[6] P. Viswanath and D. N. C. Tse, "Sum capacity of a vector Gaussian broadcast channel and uplink-downlink duality," IEEE Trans. Inf. Theory, vol. 49, pp. 1912-1921, Aug. 2003.

[7] Q. H. Spencer, A. L. Swindlehurst, and M. Haardt, "Zero-forcing methods for downlink spatial multiplexing in multiuser MIMO channels," IEEE Trans. Signal Process., vol. 52, no. 2, pp. 461-471, Feb. 2004.

[8] W. Rhee and J. M. Cioffi, "On the capacity of multiuser wireless channels with multiple antennas," IEEE Trans. Inf. Theory, vol. 49, pp. 2580-2595, Oct. 2003

[9] W. Yu, W. Rhee, S. Boyd, and J. M. Cioffi, "Iterative water filling for Gaussian vector multiple-access channels," IEEE Trans. Inf. Theory, vol. 50, no. 1, pp. 145-152, Jan. 2004.

[10] P. Gupta and P. R. Kumar, "The capacity of wireless networks," IEEE Trans. Inf. Theory, vol. 46, pp. 388-404, Mar. 2000.

[11] S. Toumpis and A. J. Goldsmith, "Capacity regions for wireless ad hoc networks," IEEE Trans. Wireless Commun., vol. 2, pp. 736-748, Jul. 2003.

[12] R. S. Blum, "MIMO capacity with interference," IEEE J. Sel. Areas Commun., vol. 21, pp. 793-801, Jun. 2003

[13] L. Zheng and D. C. Tse, "Diversity and multiplexing: A fundamental tradeoff in multiple-antenna channels," IEEE Trans. Inf. Theory, vol. 49, pp. 1073-1096, May 2003.

[14] A. B. Carleial, "Interference Channels," IEEE Trans. Inf. Theory, vol. 24, no. 1, pp. 60-70, Jan. 1978.

[15] M. H. M. Costa, "On the Gaussian interference channel," IEEE Trans. Inf. Theory, vol. 31, pp. 607-615, Sep. 1985.

[16] S. Ye and R. S. Blum, "On rate regions for wireless MIMO ad hoc networks," presented at the IEEE Vehicular Technology Conf. 2004-Fall, Los Angeles, CA, Sep. 2004.
[17] D. W. Bliss, K. W. Forsythe, A. O. Hero, and A. F. Yegulalp, "Environmental issues for MIMO capacity," IEEE Trans. Signal Process., vol. 50 no. 2, pp. 2128-2142, Feb. 2002.

[18] E. Biglieri, J. Proakis, and S. Shamai (Shitz), "Fading channels: information-theoretic and communications aspects," IEEE Trans. Inf. Theory, vol. 44, pp. 2619-2692, Oct. 1998.

[19] T. M. Cover and J. A. Thomas, Elements of Information Theory. New York: Wiley, 1991

[20] Z. D. Bai and Y. Q. Yin, "Limit of the smallest eigenvalue of a large dimensional sample covariance matrix," Ann. Probab., vol. 21, no. 3 , pp. 1275-1294, 1993.

[21] I. M. Johnstone, "On the distribution of the largest eigenvalue in principle components analysis," Ann. Statist., vol. 29, no. 2, pp. 295-327, 2001.

[22] W. C. Jakes, Microwave Mobile Communications. New York: IEEE Press, 1974

[23] G. H. Golub and C. F. Van Loan, Matrix Computations. Baltimore, MD: The Johns Hopkins Univ. Press, 1990.

Biao Chen (S'98-M'99) received the B.E. and E.E. degrees in electrical engineering from Tsinghua University, Beijing, China, in 1992 and 1994, respectively, and the M.S. degree in statistics and Ph.D. degree in electrical engineering from the University of Connecticut, Storrs, in 1998 and 1999, respectively.

From 1994 to 1995, he worked at AT\&T (China), Inc., Beijing China. From 1999 to 2000, he was a Postdoctoral Research Associate with Cornell University, Ithaca, NY. Since 2000, he has been with Syracuse University, Syracuse, NY, as an Assistant Professor with the Department of Electrical Engineering and Computer Science. He is on the editorial board of the EURASIP Journal on Wireless Communications and Networking (JWCN) and a Guest Editor for a Special Issue on Wireless Sensor Networks of EURASIP JWCN. His area of interest mainly focuses on signal processing for wireless sensor and ad hoc networks and in multiuser MIMO systems.

Michael J. Gans (S'60-M'66-SM'84-F'93) received the Ph.D. degree in electrical engineering from the University of California at Berkeley, in 1965.

Previously, he was a Member of the Wireless Communications Research Department at Bell Laboratories, Holmdel, NJ, since 1966. In 2001, he retired from Lucent Technologies. Currently, he is on the staff of AFRL/IF, Rome, NY. His primary technical areas include mobile radio, antennas, satellites, fiber optics, and infrared communications. 